DOI: http://dx.doi.org/10.35381/r.k.v4i8.274

\title{
Significatividad del marco metodológico en el desarrollo de proyectos de investigación
}

\section{Importance of the methodological framework in the development of research projects}

\author{
Ángel Enrique Azuero Azuero \\ angelazuero@hotmail.com \\ Universidad Católica de Cuenca \\ Ecuador \\ https://orcid.org/0000-0003-2176-597X
}

Recibido: 13 de octubre del 2018

Aprobado: 12 de noviembre del 2018

\section{RESUMEN}

La formulación del marco metodológico en una investigación, es permitir, descubrir los supuestos del estudio para reconstruir datos, a partir de conceptos teóricos habitualmente operacionalizados. Significa detallar cada aspecto seleccionado para desarrollar dentro del proyecto de investigación que deben ser justificado por el investigador. Respaldado por el criterio de expertos en la temática, sirviendo para responder al "como" de la investigación. Se expone el tipo de datos que se requiere buscar para dar respuesta a los objetivos, así como la debida descripción de los diferentes métodos y técnicas que se emplearan para obtener la información necesaria. Se ubicó dentro del tipo documental con un diseño bibliográfico fundamentado en la revisión sistemática del material documental. Al elegir este tipo de estudio, refieren Santa Palella y Feliberto Martins (2010) el investigador utiliza documentos, recopila, selecciona, analiza y presenta resultados coherentes.

Descriptores: Investigación; Metodología; Objetivo; Métodos; Técnicas.

\section{ABSTRACT}

The formulation of the methodological framework in an investigation, is to allow, to discover the assumptions of the study to reconstruct data, from theoretical concepts usually operationalized. It means detailing each selected aspect to be developed within 


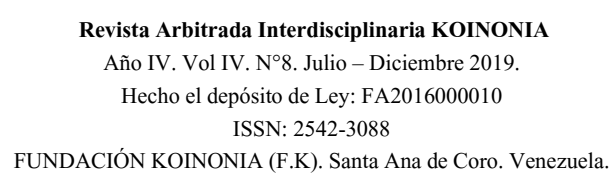

Ángel Azuero

the research project that must be justified by the researcher. Supported by the criterion of experts in the subject, serving to respond to the "how" of the investigation. The type of data that is required to search to respond to the objectives is exposed, as well as the proper description of the different methods and techniques used to obtain the necessary information. It was located within the documentary type with a bibliographic design based on the systematic review of the documentary material. When choosing this type of study, refer Santa Palella and Feliberto Martins (2010) the researcher uses documents, collects, selects, analyzes and presents consistent results.

Descriptors: Research; Methodology; Objective; Methods; Techniques

\section{INTRODUCCIÓN}

El proceso investigativo tiene como propósito final generar conocimiento a través de la resolución del problema establecido al inicio del estudio, dicho problema viene expresado normalmente, en las preguntas y objetivos de la investigación, de modo que el investigador se ve en la necesidad de tomar una serie de decisiones para enfocar el problema de investigación y buscar soluciones al mismo (Taylor y Bogdan, 1998).

La poca claridad de los enfoques del pensamiento o de la generación de teorías, deformar completamente los procesos investigativos ya que se convierten en un collage de diversos métodos que no definen su dimensión teleológica. El respecto, señala Rodríguez (2019), que:

En el devenir de la filosofía y ciencia, se ha construido diversos conocimientos en disimiles áreas del saber, constituyéndose esto en uno de los medios para la resolución de los problemas propios de la humanidad. Sin embargo, el afán desmedido del hombre por generar nuevos conocimientos a partir de los existentes o inéditos con el mismo propósito, han ocasionado algunos excesos al momento de realizar la investigación, a saber, hacen uso simultaneo de manera inconscientemente de algunos enfoques epistemológicos en el proceso investigativo, perdiendo de esta forma secuencia, claridad y concreción en los resultados del hecho investigado, además, carencia en la delimitación del enfoque filosófico y epistémico propio de la investigación (pag.264) .

En este sentido, la metodología de la investigación proporciona tanto al estudiante como a los profesionales una serie de herramientas teórico-prácticas para la solución 
de problemas mediante el método científico. Estos conocimientos representan una actividad de racionalización del entorno académico y profesional fomentando el desarrollo intelectual a través de la investigación sistemática de la realidad.

Así pues, bajo la etiqueta de "metodología de investigación" se hace referencia a todas las decisiones que el investigador toma para alcanzar sus objetivos, las cuales se enfocan en aspectos tales como el diseño de la investigación, la estrategia a utilizar, la muestra a estudiar, los métodos empleados para recoger los datos, las técnicas seleccionadas para el análisis de la información y los criterios para incrementar la calidad del trabajo, entre otras. Los diseños de investigación actuales giran en torno a dos grandes paradigmas, y no existen bases epistemológicas suficientes para establecer cual es mejor que el otro (Zapparoli, 2013). Estos son: a) El modelo "racionalista" o cuantitativo y b) El modelo "naturalista" o cualitativo.

\section{DESARROLLO TEÓRICO}

\section{MARCO METODOLOGICO}

Según revisión bibliográfica para autores como Franco (2011 p.118) el marco metodológico es el conjunto de acciones destinadas a describir y analizar el fondo del problema planteado, a través de procedimientos específicos que incluye las técnicas de observación y recolección de datos, determinando el "cómo" se realizará el estudio, esta tarea consiste en hacer operativa los conceptos y elementos del problema que estudiamos, del mismo modo, Sabino (2008 p. 25) nos dice: "en cuanto a los elementos del marco metodológico que es necesario operacionalizar pueden dividirse en dos grandes campos que requieren un tratamiento diferenciado por su propia naturaleza: el universo y las variables"

Así mismo señala Arias (2012 p.16) el marco metodológico es el "conjunto de pasos, técnicas y procedimientos que se emplean para formular y resolver problemas". Este método se basa en la formulación de hipótesis las cuales pueden ser confirmadas o descartadas por medios de investigaciones relacionadas al problema. 
De la misma manera Tamayo y Tamayo (2012 p.37) define al marco metodológico como "Un proceso que, mediante el método científico, procura obtener información relevante para entender, verificar, corregir o aplicar el conocimiento", dicho conocimiento se adquiere para relacionarlo con las hipótesis presentadas ante los problemas planteados.

Es así entonces como resumiendo las posturas de los autores puede decirse que el marco metodológico se trata en su mayoría del tercer capítulo de la tesis y es el resultado de la aplicación, sistemática y lógica, de los conceptos y fundamentos expuestos en el marco teórico. Es importante comprender que la metodología de la investigación es progresiva, por lo tanto, no es posible realizar el marco metodológico sin las fundamentaciones teóricas que van a justificar el estudio del tema elegido. Por otra parte, Marín, A; Hernández, E y Flores, J (2016) comentan que el procedimiento general planteado en la metodología como recurso didáctico para emprender la teorización es cíclico, de acción participativa y de evaluación constante entre los investigadores y los sujetos de estudio.

\section{Investigación cuantitativa}

Según el modelo "racionalista" o cuantitativo, la ciencia surge como una necesidad del ser humano por aprender sobre los fenómenos que ocurren a su alrededor y sus relaciones de causa y efecto, con el fin de poder interferir en ellos o utilizar este conocimiento a su favor. Según Jensen (2009), la unidad de la ciencia se refiere a tres aspectos:

1) Unidad del lenguaje: todos los enunciados científicos deben satisfacer los requerimientos particulares del lenguaje de la física $y$, en consecuencia, toda observación científica debe conducir a la mensurabilidad de los fenómenos estudiados.

2) Unidad de las leyes: los hechos pueden ser explicados siguiendo principios similares.

3) Unidad del método: en la actualidad este aspecto es el que encuentra más apoyo cuando se aparta de la definición original del método científico (procedimientos de medición y evaluación), para convertirse en un concepto que se relaciona más bien con 
las formas y los medios con que se fundamentan las "pretensiones de validez". Basados en las dos argumentaciones iniciales (unidad de lenguaje y unidad de leyes científicas), es comprensible que exista una clara preferencia por los métodos cuantitativos de investigación.

Asimismo, Chavarría, (2011) comenta que está tan arraigado el vínculo entre ciencia, método científico y cuantificación que es difícil incluso percatarse de su repercusión sobre el desarrollo de la praxis y la tendencia de otorgarle mayor credibilidad. Sin embargo, estos dos pilares del empirismo lógico, muy apropiados para las ciencias exactas, son inconvenientes en las ciencias sociales donde la acumulación de hechos no es suficiente para explicar fenómenos socioculturales.

Como bien lo indica Jensen (2009), cada vez más se busca el "pluralismo complementario" donde la verdad es investigada por grupos interdisciplinarios, para los cuales es difícil unificar el lenguaje o las leyes. La metodología cuantitativa de investigación se caracteriza según Bryman, (1988):

- En ella el contacto del investigador con el sujeto objeto de estudio es prácticamente nulo, dado que el investigador cuantitativo mantiene este distanciamiento, su postura respecto al sujeto de estudio es la de un intruso, la de un desconocido que aplica un marco de trabajo establecido a priori sobre el objeto de su investigación y que se implica lo menos posible en el contexto social donde se desarrolla el fenómeno a ser estudiado.

- Las teorías y conceptos existentes suponen el punto de partida para la investigación, siendo el objetivo de esta última el de confirmar o no la veracidad de dichas teorías y conceptos.

- En ella se tiende a adoptar un enfoque estructurado para estudiar el fenómeno, siendo esta estructura consecuencia, en gran parte, de las técnicas utilizadas para recoger la información.

- Posibilita inferir los hallazgos de la investigación en poblaciones más grandes, esto es, permite la generalización estadística de los resultados. 
- Transmite una visión de la realidad social que es estática como consecuencia de que tiende a descuidar el impacto y papel del cambio en la vida social.

- Los investigadores cuantitativos ven la realidad social como externa a los sujetos y como una fuerza que les condiciona.

- Los datos que emanan de investigaciones cuantitativas son considerados, a menudo, como tangibles, rigurosos y fidedignos, dichos adjetivos sugieren que estos datos poseen una precisión considerable, han sido recogidos a través de procedimientos sistemáticos y pueden ser fácilmente contrastados por otro investigador.

Así pues, las características descritas anteriormente hacen que la metodología cuantitativa sea más conveniente cuando exista un cuerpo teórico previo suficientemente fundamentado capaz de definir los conceptos a analizar de forma concreta y mensurable (Miquel et al., 1997; Saunders et al., 2009).

Esto implica que la metodología cuantitativa se presenta como más apropiada para la verificación o el contraste de hipótesis fundamentadas en el conocimiento teórico existente que para construir o avanzar en la formación de una teoría todavía en fase de desarrollo (Flick, 2009; gill y Johnson, 2010).

Así mismo, la metodología cuantitativa no se centra en explorar, describir o explicar, un único fenómeno, sino que busca realizar inferencias a partir de una muestra hacia una población, evaluando para ello la relación existente entre aspectos o variables de las observaciones de dicha muestra (Gauri y Gronhaug, 2010; Ragin, 1999), además de emplear categorías predeterminadas que permitan someter los datos obtenidos a análisis estadístico (Skinner et al., 2010). Así, el objetivo de la investigación cuantitativa pasa a ser la identificación de patrones generales que caracterizan a la totalidad de una población.

\section{Investigación cualitativa}

En relación a este tipo de investigación se quiso hacer una referencia un poco más amplia y detallada, por ser la seleccionada como investigador para hacer el proyecto de tesis doctoral, esto por considerarla la más apropiada para poder abordar la temática de 
estudio y por precisamente utilizar la entrevista como instrumento de recolección de información a los sujetos clave y que al mismo tiempo forma parte de los elementos del marco metodológico en una investigación cualitativa.

El estudio de los modelos, es complicado, puesto que no hay un modelo puro, sin embargo, la energía que se aplique para su reconocimiento, es positivo, desde la idea que favorece a unos como a otros, la comprensión sobre las distintas maneras a través de lograr los objetivos, tarea que, además, sobrelleva la disputa de cómo se piensa el conocimiento, desde las distintas formas de relatar el suceso observado, creando el modelo representacional, en consecuencia el modelo epistémico.

A la par, en cuanto propósito del enfoque epistémico, Padrón (2011), indica que su objeto, es la explicación del saber, de los significados coligados, orígenes, reflexiones, tipos y correspondencia existente, entre el investigador y el suceso conocido. Según lo indicado, lo que busca, es verificar la naturaleza las opiniones científicas, la afirmación de cada investigador con la situación real, cómo la ciencia representa, expresa, expone, ayudando al control de esa naturaleza y uso del método científico, igualmente los distintos razonamientos que se utilizan para instituir conclusiones como alcances de los distintos métodos y modelos de ciencia.

De acuerdo con lo anterior, la metodología cualitativa de investigación parte de fundamentos y premisas radicalmente distintas a las establecidas para los métodos cuantitativos; tal como afirma Zapparoli (2013, pág. 194): los positivistas adoptan como modelo de investigación el tomado de las ciencias naturales, buscan el conocimiento de las causas mediante métodos como cuestionarios, inventarios y estudios demográficos que le permiten el análisis estadístico.

Los métodos cualitativos son paradigmas, aplicados comúnmente en las ciencias sociales, donde los fenómenos no se pueden comprender en toda su amplitud desde información cuantitativa. Como Vélez de la Calle (2009) sostiene, los ensayos basados en historias de vida se preguntan de qué manera y por qué la información suministrada por la historia biográfica de una persona puede generar conocimiento, a lo cual se 
responde con que la cultura se construye en la mente y las acciones de esas personas.

Es decir, los enfoques cualitativos sirven para comprender la realidad social, porque dejan de lado las visiones unificadas que no se pueden aplicar al hecho social donde no hay leyes generalizadas, sino sentimientos, pensamientos e historias de los actores sociales que son captados a través de sus testimonios.

La investigación cualitativa atraviesa diversas disciplinas, participa de una gran variedad de discursos o perspectivas teóricas y engloba numerosos métodos y estrategias de recogida de datos. Esta riqueza denota la complejidad y alcance del enfoque cualitativo en el abordaje de la investigación socioeducativa y requiere que se ensayen clasificaciones o categorías que aporten un orden conceptual en el ámbito investigación y permitan la comunicación en la comunidad investigadora.

Sin embargo, y paradójicamente, cuando se realiza un análisis de las diversas tipologías de modalidades de investigación cualitativa se constata la existencia de una pluralidad de términos usados con diferentes sentidos, que en ocasiones se convierte en una maraña conceptual desconcertante, y que lejos de simplificar esta cuestión, dificulta la tarea de establecer un marco común y homogéneo de clasificación.

Varios autores plantean diversas tipologías de los diseños cualitativos, particularmente en el presente trabajo nos basaremos en la que proponen Hernández, Fernández \& Baptista (2014) que, aunque no abarca todos los marcos interpretativos, si comprende los principales. Nos referimos a los diseños de teoría fundamentada, diseños etnográficos, diseños narrativos, diseños de investigación-acción y diseños fenomenológicos.

Para Chavarría, (2011) el fenomenólogo, busca la comprensión de los hechos mediante métodos cualitativos que le proporcionen un mayor nivel de comprensión de los motivos y creencias que están detrás de las acciones de las personas. El lenguaje científico se fundamenta en la demostración objetiva de la causalidad lineal, pero ya se ha demostrado que no existe una separación sujeto-objeto, ya que las condiciones de 
observación (escogidas por el investigador) siempre determinan lo observado. Sistematizar resultados en entornos vivos, donde hay consecuencias de la indagación para las personas involucradas.

En el mismo sentido, comenta el citado autor, es un proceso más difícil, largo e incierto que los de los estudios cuantitativos donde no se requiere ese grado de sensibilidad que exigen los estudios sociales. Los estudios cualitativos se prefieren por sus propiedades explicativas y su poder exploratorio ayuda a esclarecer los resultados obtenidos en investigaciones cuantitativas o a generar teorías (que más tarde se deben de confirmar con los métodos cuantitativos) en campos poco explorados.

No obstante, es importante distinguir que no existe una clara separación entre ellos, ya que la mayoría de los estudios toma elementos de más de uno de éstos, es decir, se yuxtaponen. Por eso con mucha frecuencia se afirma que las fronteras entre los diseños cualitativos realmente no existen. Por ejemplo, un estudio orientado por la teoría fundamentada puede abarcar elementos narrativos y fenomenológicos. A continuación, revisaremos brevemente cada uno de ellos.

\section{a) Diseños de Teoría Fundamentada:}

La teoría fundamentada según Glaser \& Strauss, (1967) se basa en el interaccionismo simbólico. Su planteamiento básico es que las proposiciones teóricas surgen de los datos obtenidos en la investigación, más que de los estudios previos. Es el procedimiento el que genera el entendimiento de un fenómeno educativo, psicológico, comunicativo o cualquier otro que sea concreto.

Para Creswell, (2005) la teoría fundamentada es especialmente útil cuando las teorías disponibles no explican el fenómeno o planteamiento del problema, o bien, cuando no cubren a los participantes o muestra de interés. La teoría fundamentada va más allá de los estudios previos y los marcos conceptuales preconcebidos, en búsqueda de nuevas formas de entender los procesos sociales que tienen lugar en ambientes naturales. Este tipo de diseños se pueden clasificar en diseños sistemáticos y diseños emergentes. 


\section{b) Diseños Etnográficos:}

Estos diseños buscan describir y analizar ideas, creencias, significados, conocimientos y prácticas de grupos, culturas y comunidades. Incluso pueden ser muy amplios y abarcar la historia, la geografía y los subsistemas socioeconómico, educativo, político y cultural de un sistema social (rituales, símbolos, funciones sociales, parentesco, migraciones, redes, entre otros).

Alvarez-Gayou (2013) considera que el propósito de la investigación etnográfica es describir y analizar lo que las personas de un sitio, estrato o contexto determinado hacen usualmente; así como los significados que le dan a ese comportamiento realizado bajo circunstancias comunes o especiales, y presentan los resultados de manera que se resalten las regularidades que implica un proceso cultural.

Por su parte, Creswell (Citado por Hernández, Fernández \& Baptista, 2014) señala que los diseños etnográficos estudian categorías, temas y patrones referidos a las culturas. El investigador reflexiona sobre ¿Qué cualidades posee el grupo o comunidad que lo(a) distinguen de otros(as)?, ¿Cómo es su estructura?, ¿Qué reglas regulan su operación?, ¿Qué creencias comparten?,

¿Qué patrones de conducta muestran?, ¿Cómo ocurren las interacciones?, ¿Cuáles son sus condiciones de vida, costumbres, mitos y ritos?, ¿Qué procesos son centrales para el grupo o comunidad?, entre otros. En el mismo sentido, Denis \& Gutiérrez (Citado por Bernal, 2009) el rigor de los estudios etnográficos está dado por las reconstrucciones teóricas, y por la búsqueda de coherencia entre las interpretaciones y la realidad estudiada.

Dentro de este tipo de diseños, existen varias clasificaciones. Por ejemplo, Creswell (2005) los divide en: (1) Diseños realistas o mixtos; (2) Diseños críticos; (3) Diseños clásicos; (4) Diseños microetnográficos y (5) Estudios de casos culturales. Por su parte Boyle (Citado por Alvarez-Gayou, 2013) basándose en el tipo de unidad social estudiada los divide en: (1) Etnografias procesales; (2) Etnografía holística o clásica; (3) 
Etnografía particularista; (4) Etnografía de corte transversal; y (5) Etnografía etnohistórica.

\section{c) Diseños Narrativos:}

En los diseños narrativos el investigador recolecta datos sobre las historias de vida y experiencias de determinadas personas para describirlas y analizarlas. Son de interés las personas en sí mismas y su entorno. Creswell (2005) señala que el diseño narrativo en diversas ocasiones es un esquema de investigación, pero también es una forma de intervención, ya que el contar una historia ayuda a procesar cuestiones que no estaban claras. Se usa frecuentemente cuando el objetivo es evaluar una sucesión de acontecimientos.

Los datos se obtienen de autobiografías, biografías, entrevistas, documentos, artefactos y materiales personales y testimonios (que en ocasiones se encuentran en cartas, diarios, artículos en la prensa, grabaciones radiofónicas y televisivas, entre otros). Pueden referirse a: (1) Toda la historia de la vida de una persona o grupo; (2) Un pasaje o época de dicha historia de vida o (3) Uno o varios episodios.

El investigador analiza diversas cuestiones: la historia de vida, pasaje 0 acontecimiento(s) en sí; el ambiente (tiempo y lugar) en el cual vivió la persona o grupo, o sucedieron los hechos; las interacciones, la secuencia de eventos y los resultados. En este proceso, el investigador reconstruye la historia de la persona o la cadena de sucesos (casi siempre de manera cronológica: de los primeros hechos a los últimos), posteriormente los narra bajo su óptica y describe (sobre la base de la evidencia disponible) e identifica categorías y temas emergentes en los datos narrativos (que provienen de las historias contadas por los participantes, los documentos, materiales y la propia narración del investigador).

Así también Mertens (2005) divide a los estudios narrativos en: (1) De tópicos (enfocados en una temática, suceso o fenómeno); (2) Biográficos (De una persona, grupo o comunidad; sin incluir la narración de los participantes "en vivo", ya sea porque fallecieron o no recuerdan a causa de su edad avanzada o enfermedad, o son 
inaccesibles); (3) Autobiográficos (de una persona, grupo o comunidad incluyendo testimonios orales "en vivo" de los actores participantes).

\section{d) Diseños de Investigación-Acción:}

La finalidad de la Investigación-Acción es resolver problemas cotidianos e inmediatos, y mejorar prácticas concretas. Su propósito fundamental se centra en aportar información que guíe la toma de decisiones para programas, procesos y reformas estructurales. Los pilares sobre los cuales se fundamentan los diseños de investigación-acción son:

- Los participantes que están viviendo un problema son los que están mejor capacitados para abordarlo en un entorno naturalista.

- La conducta de estas personas está influida de manera importante por el entorno natural en que se encuentran.

- La metodología cualitativa es la mejor para el estudio de los entornos naturalistas.

Según Stringer (Citado por Hernández, Fernández \& Baptista, 2014) las tres fases esenciales de los diseños de investigación-acción son: Observar (construir un bosquejo del problema y recolectar datos), pensar (analizar e interpretar) y actuar (resolver problemas e implementar mejoras), las cuales se dan de una manera cíclica, una y otra vez, hasta que el problema es resuelto, el cambio se logra o la mejora se introduce satisfactoriamente. Para Creswell (2005) divide a los diseños fundamentales de la investigación-acción en dos clases: Práctico y Participativo.

\section{e) Diseños Fenomenológicos:}

Estos diseños se enfocan en las experiencias individuales subjetivas de los participantes. Responden a la pregunta ¿Cuál es el significado, estructura y esencia de una experiencia vivida por una persona (individual), grupo (grupal) o comunidad (colectiva) respecto de un fenómeno? El centro de indagación de estos diseños reside en la(s) experiencia(s) del participante o participantes. 
De acuerdo con Creswell, 2005; Alvarez-Gayou, 2013; y Mertens, 2005 (Citado por Hernández, Fernández \& Baptista, 2014) la fenomenología se fundamenta en las siguientes premisas:

- Se pretende describir y entender los fenómenos desde el punto de vista de cada participante y desde la perspectiva construida colectivamente.

- Se basa en el análisis de discursos y temas específicos, así como en la búsqueda de sus posibles significados.

- El investigador confía en la intuición y en la imaginación para lograr aprehender la experiencia de los participantes.

- El investigador contextualiza las experiencias en términos de su temporalidad (tiempo en que sucedieron), espacio (lugar en el cual ocurrieron), corporalidad (las personas físicas que la vivieron), y el contexto relacional (los lazos que se generaron durante las experiencias).

- Las entrevistas, grupos de enfoque, recolección de documentos y materiales e historias de vida se dirigen a encontrar temas sobre experiencias cotidianas y excepcionales.

\section{A MANERA DE REFLEXIONES}

a. El método elegido en una investigación afecta los aciertos ya por extensión, la interpretación. La metodología es crucial porque un método poco confiable produce resultados poco confiables, socavando su valor.

b. Es necesaria la explicación de cómo se obtuvieron e interpretaron estos resultados. En eso consiste el marco metodológico.

c. Este marco posee ciertas características distintivas. Primeramente, en esta sección se debe dar una explicación detallada del estudio. Es muy importante en la ciencia que los resultados sean replicables.

d. Si los autores proporcionan suficientes detalles, otros científicos pueden repetir sus experimentos para verificar sus hallazgos. 
e. Esta información es particularmente importante cuando se ha desarrollado un nuevo método o se utiliza un uso innovador de un método existente.

f. En la mayoría de los casos, hay una variedad de métodos diferentes para dar respuesta a un problema de investigación. Además, existen distintas técnicas y procesos ampliamente aceptados en cada campo de estudio

g. En el marco metodológico se deben exponer claramente las razones por las que se eligió un procedimiento o técnica en particular.

h. Tiene que dejar constancia de que los datos fueron recopilados o generados de una manera consistente con la práctica aceptada en las distintas disciplinas.

i. Además, se debe tomar en cuenta que el marco metodológico es un texto del género científico. Por tanto, la redacción debe ser directa y ordenada. Generalmente se escribe en voz pasiva y en tercera persona.

j. Sin embargo, el paradigma cualitativo acepta voz activa y primera persona. Para mayor claridad, cuando se debe presentar una gran cantidad de detalles, la información se debe presentar en subsecciones según el tema. El material en cada sección debe organizarse por tema de mayor a menor importancia.

\section{REFERENCIAS CONSULTADAS}

1. Alvarez-Gayou, J. (2013). Cómo hacer investigación cualitativa: Fundamentos y Metodología. México: Paidós.

2. Arias, F. (2012). Proyecto de investigación: introducción a la metodología científica $\left(5^{\circ}\right.$ ed.) Caracas: Espíteme.

3. Bernal, C. (2009). Metodología de la Investigación. México: Prentice Hall.

4. Bryman, A. (1988). Quantity and Quality in social research, Unwin hyman, London.

5. Chavarría g., M. (2011). La dicotomía cuantitativa / cualitativo: falsos dilemas en investigación social. Actualidades en Psicología. 25, 1-35. 
6. Creswell, J. (2005). Educational research: Planning, conducting, and evaluating quantitative and qualitative research. Upper Saddle River: Pearson Education.

7. Flick, U. (2009). An Introduction to Qualitative. Research. Cuarta edición. SAGE Publications. Londres.

8. Franco, Y (2011) Tesis de Investigación. Marco Metodológico. Venezuela. Disponible en: Disponible: http://tesisdeinvestig.blogspot.com/2011/06/marcometodologico- definicion.html [Consulta 2018/11/22].

9. Gill, J. y Johnson P. (2010). Research Methods for Managers. Cuarta edición. SAGE Publications. Londres.

10. Glaser, B. \& Strauss, A. (1967). The Discovery of Grounded Theory: Strategies for Qualitative Research. Chicago: Aldine.

11. Hernández, R., Fernández, C. \& Baptista, P. (2014). Metodología de la Investigación. México: Mc Graw Hill.

12. Jensen, h. (2009). La investigación interdisciplinaria. girasol: revista de la escuela de estudios generales. 1, 41-50.

13. Marín, A; Hernández, E y Flores, J (2016) Metodología para el análisis de datos cualitativos en investigaciones orientadas al aprovechamiento de fuentes renovables de energía. Revista Arbitrada KOINONIA. Vol. 1, Núm. 1 (1): Enero Junio. 2016.

14. Mertens, D. (2005). Research and evaluation in Education and Psychology: Integrating diversity with quantitative, qualitative, and mixed methods. Thousand Oaks: Sage.

15. Miquel, S., Bigné, E., Lévy, J. P., Cuenca, A. C. \& Miquel, M. J. (1997): Investigación de mercados, Mcgraw-hill, Madrid.

16. Padrón, J. (2011). Paradigmas de investigación en Ciencias Sociales. Un enfoque curricular. Disponible en http://padron.entretemas.com/paradigmas.htm.

17. Rodríguez, A. (2019). Desde la filosofía hacia el pensamiento emergente en el desarrollo de los procesos investigativos. Revista Arbitrada Interdisciplinaria Koinonía, 4(7), 262-279. Recuperado de http://fundacionkoinonia.com.ve/ojs/index.php/revistakoinonia/article/view/204 
18. Saunders, M., Lewis, P. \& Thornhill, A. (2009). Research methods for business students, Prentice Hall, Harlow (Essex).

19. Skinner, D., Tagg, C. \& Holloway, J. (2010) Managers and Research. The Pros and Cons of Qualitative Approaches. Management Learning, 31(2), 163-179.

20. Tamayo y Tamayo. (2012) El Proceso de la Investigación Científica. Limusa Noriega Editores. 4ta Edición. México.

21. Taylor, S. y R.C. Bogdan (1998): Introducción a los métodos cualitativos de investigación. Paidós, Barcelona.

22. Vélez de la Calle, C. (2009). Las historias de vida, fundamentos y metodologías para la investigación educativa y social. Revista fundación universitaria Luis amigó. Colombia.

23.Zapparoli, M. (20013). Concepciones teóricas metodológicas sobre investigación. Girasol: revista de la escuela de estudios generales. 5, 191198.Todos los derechos reservados. Universidad de Costa Rica.

\section{CONSULTED REFERENCE}

1. Alvarez-Gayou, J. (2013). How to do qualitative research: Fundamentals and Methodology. Mexico: Paidós.

2. Arias, F. (2012). Research project: introduction to scientific methodology (5th ed.) Caracas: Espíteme.

3. Bernal, C. (2009). Investigation methodology. Mexico: Prentice Hall.

4. Bryman, A. (1988). Quantity and Quality in social research, Unwin hyman, London.

5. Chavarría g., M. (2011). The quantitative / qualitative dichotomy: false dilemmas in social research. News in Psychology. 25, 1-35.

6. Creswell, J. (2005). Educational research: Planning, conducting, and evaluating quantitative and qualitative research. Upper Saddle River: Pearson Education. 
7. Flick, U. (2009). An Introduction to Qualitative. Research. Fourth edition. SAGE Publications. London.

8. Franco, Y (2011) Research Thesis. Methodological framework. Venezuela. Available at: Available: http://tesisdeinvestig.blogspot.com/2011/06/marcometodologico-defunción.html [Consulta 2018/11/22].

9. Gill, J. and Johnson P. (2010). Research Methods for Managers. Fourth edition. SAGE Publications. London.

10. Glaser, B. \& Strauss, A. (1967). The Discovery of Grounded Theory: Strategies for Qualitative Research. Chicago: Aldine.

11. Hernández, R., Fernández, C. \& Baptista, P. (2014). Investigation methodology. Mexico: Mc Graw Hill.

12. Jensen, h. (2009). The interdisciplinary research. sunflower: magazine of the school of general studies. 1, 41-50.

13. Marine; Hernández, E and Flores, J (2016) Methodology for the analysis of qualitative data in research aimed at the use of renewable energy sources. KOINONIA Arbitrated Magazine. Vol. 1, No. 1 (1): January - June. 2016

14. Mertens, D. (2005). Research and evaluation in Education and Psychology: Integrating diversity with quantitative, qualitative, and mixed methods. Thousand Oaks: Sage.

15. Miquel, S., Bigné, E., Lévy, J. P., Cuenca, A. C. \& Miquel, M. J. (1997): Market research, Mcgraw-hill, Madrid

16. Padrón, J. (2011). Paradigms of research in Social Sciences. A curricular approach Available at http://padron.entretemas.com/paradigmas.htm.

17. Rodríguez, A. (2019). From philosophy to emerging thinking in the development of research processes. Journal Interdisciplinary Arbitrated Koinonía, 4 (7), 262279. http://fundacionkoinonia.com.ve/ojs/index.php/revistakoinonia/article/view/204

18. Saunders, M., Lewis, P. \& Thornhill, A. (2009). Research methods for business students, Prentice Hall, Harlow (Essex). 
19. Skinner, D., Tagg, C. \& Holloway, J. (2010) Managers and Research. The Pros and Cons of Qualitative Approaches. Management Learning, 31 (2), 163-179.

20. Tamayo and Tamayo. (2012) The Process of Scientific Research. Limusa Noriega Editors. 4th Edition Mexico.

21. Taylor, S. and R.C. Bogdan (1998): Introduction to qualitative research methods. Paidós, Barcelona.

22. Vélez de la Calle, C. (2009). The life stories, foundations and methodologies for educational and social research. Luis amigó university foundation magazine. Colombia.

23.Zapparoli, M. (20013). Methodological theoretical concepts about research. Sunflower: magazine of the school of general studies. 5, 191-198. All rights reserved. Costa Rica university. 